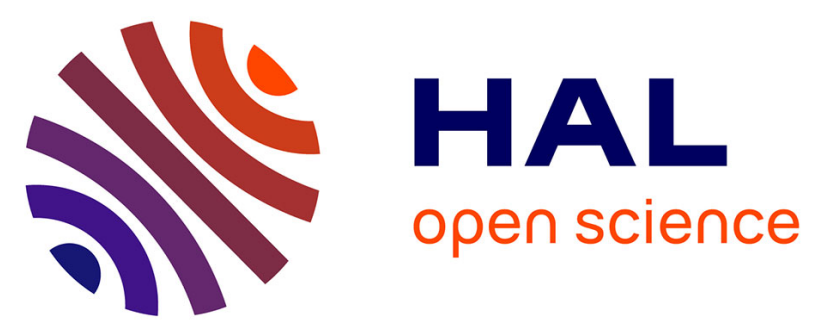

\title{
LONGITUDINAL AND TRANSVERSE COILED GIANT MAGNETOIMPEDANCE TRANSDUCERS PRINCIPLE, MODELING AND PERFORMANCES
}

Joël Moutoussamy, Christophe Coillot, Gérard Chanteur, Francisco Alves

\section{- To cite this version:}

Joël Moutoussamy, Christophe Coillot, Gérard Chanteur, Francisco Alves. LONGITUDINAL AND TRANSVERSE COILED GIANT MAGNETOIMPEDANCE TRANSDUCERS PRINCIPLE, MODELING AND PERFORMANCES. Transducers'09, Jun 2009, Denver, United States. pp.M3P.076. hal-00694531

\section{HAL Id: hal-00694531 \\ https://hal.science/hal-00694531}

Submitted on 4 May 2012

HAL is a multi-disciplinary open access archive for the deposit and dissemination of scientific research documents, whether they are published or not. The documents may come from teaching and research institutions in France or abroad, or from public or private research centers.
L'archive ouverte pluridisciplinaire HAL, est destinée au dépôt et à la diffusion de documents scientifiques de niveau recherche, publiés ou non, émanant des établissements d'enseignement et de recherche français ou étrangers, des laboratoires publics ou privés. 


\title{
LONGITUDINAL AND TRANSVERSE COILED GIANT MAGNETOIMPEDANCE TRANSDUCERS PRINCIPLE, MODELING AND PERFORMANCES
}

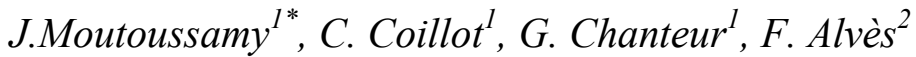 \\ ${ }^{1}$ LPP, 10-12, Avenue de 1'Europe, 78140 Vélizy, FRANCE \\ ${ }^{2}$ LGEP, 11 rue Joliot-Curie, Plateau du Moulon, 91192 Gif sur Yvette, FRANCE
}

\begin{abstract}
New Giant Magneto-Impedance structures are studied both experimentally and theoretically. These transducers are made with one or two anisotropic nanocrystalline ribbons and the magnetic excitation is provided by coils mounted either in transverse or longitudinal direction. Very high sensitivities up to $1000 \mathrm{~V} / \mathrm{T}$ have been achieved even at low excitation frequencies from $5 \mathrm{kHz}$ to $500 \mathrm{kHz}$ and low consumption driving current (5-10mA). The Eddy current phenomenon and magnetic domain considerations are used to determine a semi-theoretical model of impedance. Intrinsic sensitivity computation approach emphasizes on the role of the differential magnetic permeability in coiled GMI detection performances.
\end{abstract}

\section{KEYWORDS}

Coiled GMI, intrinsic sensitivity, differential magnetic permeability,

\section{INTRODUCTION}

Since the work of Harrison et al [1] on the Eddy current in ferromagnetic wire modified by a DC magnetic field, the GMI effect has been the subject of many experimental and theoretical studies. In those studies [1-2], the authors have reported the physics background considering the dispersion of electromagnetic waves inside magnetic wire and sandwich transducer. By using micro magnetic theory the susceptibility tensor has been deduced in the case of the anisotropic magnetic core and combined with the impedance expression where the skin effect is often considered to be the main element responsible of the GMI ratio. From these works, fundamental analysis criteria were brought out such as the GMI ratio, the anisotropic magnetic $H_{k}$ field and the AC permeability in the case of the direction configuration corresponding to the $\mathrm{AC}$ field perpendicular to the DC field. In this configuration, the impedance curve shows even parity form with the DC field and resonant magnetic field appears at $H_{k}$. However, odd curve impedance was also obtained in anisotropic material [3] by measuring the induced voltage through a pick-up coil in a GMI sandwich while the excitation was connected to the end of the copper conductor inside it. In this context, coiled GMI transducers benefits from the two components of the diagonal differential magnetic permeability components by using longitudinal or transverse coils [4]. One of the main advantages of coils is, for an identical excitation current, to increase the magnetic excitation and finally the intrinsic sensitivity $S_{\text {int }}$ which is the relevant parameter for weak magnetic field measurements [5].
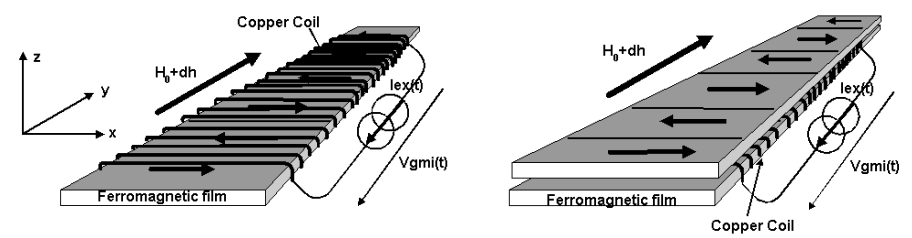

Fig 1: GMI with one and two ferromagnetic ribbons and a transverse coil.
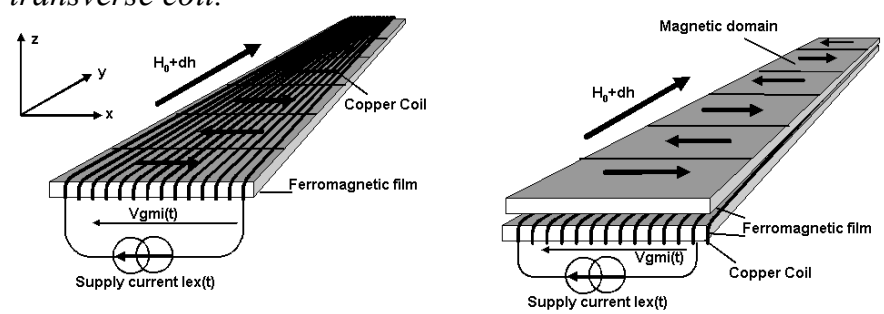

Fig 2: GMI with one and two ferromagnetic ribbons and a longitudinal coil.

By considering that the coiled GMI, with conductive ferromagnetic ribbon, exploits only self-induction behaviour (without eddy current because of low frequencies driving current), we express the coil output signal as an auto-induced voltage as written in relation (1). In this expression $R_{0}$ is the air reluctance of the coil, $\mu_{\text {diff }}$ represents the differential magnetic permeability at the DC field coordinate $H_{0}$ and $I_{e x c}$ is the driving current.

$\underline{V}_{g m i}=j \omega \mathrm{R}_{0}\left(\mu_{d i f f}\left(H_{0}\right)+\left(\frac{\partial \mu_{d i f f}}{\partial H_{e x t}}\right)_{H_{0}} d b\right) \underline{I}_{e x}$

By writing (1) we assume that the measured magnetic field $d h$ is proportional to the derivative of differential permeability. Relation (1) reminds the signal output of Search Coil and Fluxgate sensors. Nevertheless, a difference is noticeable from the first type because the DC field is naturally undetectable by a coil and also from the second type because coiled GMI have linear working behavior from the driving current. Thus, the coiled GMI principle is a part of the differential magnetic permeability variation described by R.M. Bozorth [6] as the so-called reversible or incremental permeability. To confirm this principle and determine optimum parameters, experimental studies have been done by measuring impedance and intrinsic sensitivity in low frequency range and DC field 
range from $0 \mathrm{~A} / \mathrm{m}$ to $H_{\max }=2100 \mathrm{~A} / \mathrm{m}$. However, the experimental investigations presented are limited to the transverse coiled with one ribbon and the longitudinal coiled with two ribbons. In the purpose of the magnetometer design it is convenient to have an analytic formula for the impedance and the intrinsic sensitivity. Then, from the experimental studies, the differential magnetic permeability has been deduced for the transverse sample and the micro magnetic approach has confirmed the model presented.

\section{EXPERIMENT}

These transducers have identical magnetic ribbon of $20 \mu \mathrm{m}$ thick, $2 \mathrm{~cm}$ wide and $10 \mathrm{~cm}$ long and the coils both have 60 turns of insulated copper wounded around the magnetic layer. The experimental work consists in measuring intrinsic sensitivity rather than the MI ratio by using a power spectrum analyzer with an AM modulation method measurement [5] that provides a direct measurement of the intrinsic sensitivity. Then a solenoid coil was used to generate the DC field $H_{0}$ and a pair of Helmholtz coils to produce the low frequency magnetic field $d h$ of $1.2 \mu \mathrm{T}$. Phase modulation also occurs in output signal $V_{g m i}(t)$ but it remains less than amplitude modulation in this frequency range.

\section{RESULTS}

\section{Effect of the longitudinal DC field}

Figure 3 and Figure 4 show impedance results from the transverse and longitudinal coil. As sandwich GMI, coiled GMI impedance exhibit even parity curve but the decrease with respect to the DC field is greatly pronounced. The experimental MI ratios are, respectively, approximately $433 \%$ and $666 \%$ while maximum intrinsic sensitivities are $0.17 \mathrm{Ohms} / \mathrm{A} / \mathrm{m}$ and $0.1 \mathrm{Ohms} / \mathrm{A} / \mathrm{m}$ (Figure 5 and Figure 6). Thus, MI ratio provides information on strong magnetic field behaviour while intrinsic sensitivity provides information on weak magnetic field behaviour. Careful examination of intrinsic sensitivity curve shows that the greatest value of $S_{\text {int }}$ occurs at the same $H_{0}$ coordinate and just at the beginning of the decrease of the impedance, resulting in the maximum derivative of magnetic permeability being located at this point. This coordinate corresponds to the anisotropic magnetic field $H_{k}$ which is related to the ribbon shape with respect to the DC field and the magneto-crystalline anisotropy. Moreover, the two coiled GMI show different impedance $Z\left(H_{0}\right)$ curve shape, in particular a monotonous decrease for the transverse GMI which does not exhibit peak impedance as the longitudinal GMI does. These different behaviours suggest that only uniform magnetization rotation occurs in the transverse configuration whereas for longitudinal configuration, the walls' displacement dictates magnetization below $H_{k}$ and rotation above it.

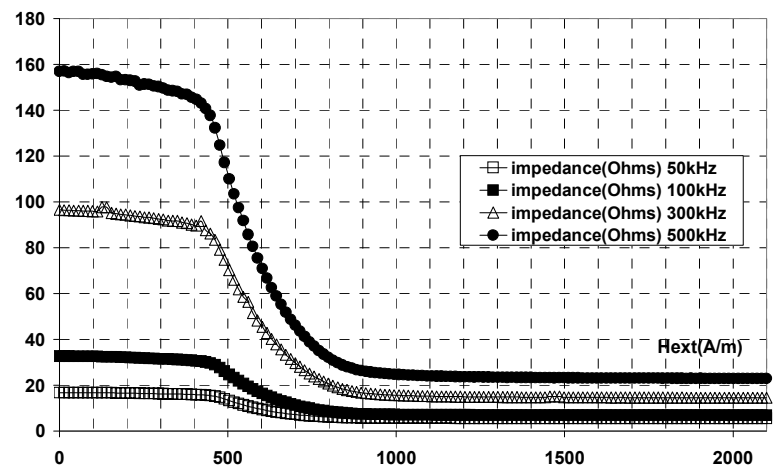

Fig 3: Transverse coiled GMI with one ribbon: Impedance with $D C$ field at different frequencies.

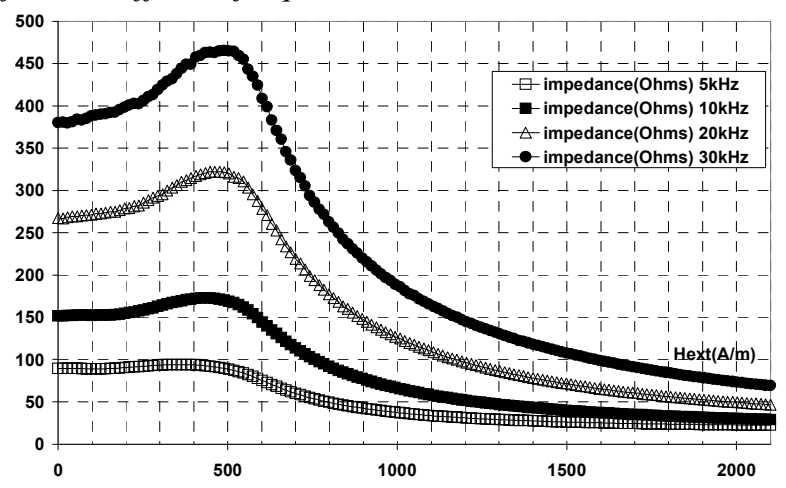

Fig 4: Longitudinal coiled GMI with two ferromagnetic ribbons: Impedance with DC field at different frequencies.

\section{Effect of the electric current frequency}

Owing to inductive behaviour, impedance $Z(f)$ and intrinsic sensitivity $S_{\text {int }}(f)$ change with a linearity curve until $f=200 \mathrm{kHz}$ for a DC field equal to $H_{k}$ (Figure 7). As frequency increases, intrinsic sensitivity (Figure 8) reaches its maximum value at $800 \mathrm{kHz}$ and begins to decrease at $1 \mathrm{MHz}$. The impedance becomes less inductive but more resistive. Eddy currents are developing more and more and GMI becomes less sensitive in relation to the weak DC field (Figure 9). However, strong DC field $H_{\max }$ removes the intrinsic sensitivity and the impedance becomes again inductive which means Eddy currents are removed [2] inside the magnetic layer because of the decrease of the magnetic permeability. Although that maximum intrinsic sensitivity has been reached at high frequency, the equivalent sensitivity $S_{\text {equi }}$ defined as the product of $S_{\text {int }}$ by $\mathrm{I}_{\mathrm{ex}}$ has been obtained at a low enough frequency in order to diminish the impedance and thus increase the electric current. Finally, at a frequency equal to $300 \mathrm{kHz}$ the transverse coiled GMI exhibits magnetic induction sensitivity as high as $1000 \mathrm{~V} / \mathrm{T}\left(\mathrm{I}_{\mathrm{ex}}=8 \mathrm{~mA}\right.$ and $\left.\mathrm{H}_{0}=500 \mathrm{~A} / \mathrm{m}\right)$ without electronic amplifiers. In comparison to the $0.7 \mathrm{~V} / \mathrm{T}$ $(\mathrm{Iex}=1 \mathrm{~mA})$ maximum sensitivity of the Hall sensor [7]. 


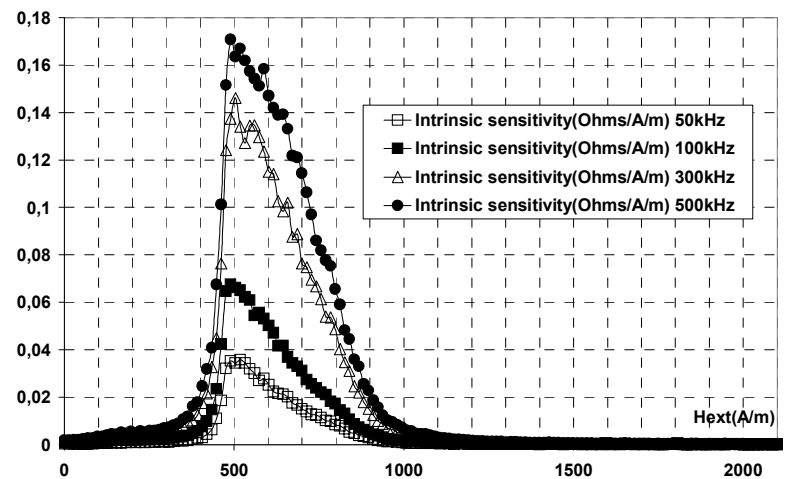

Fig 5: Transverse coiled GMI with one ribbon: Intrinsic sensitivity with DC field at different frequencies.

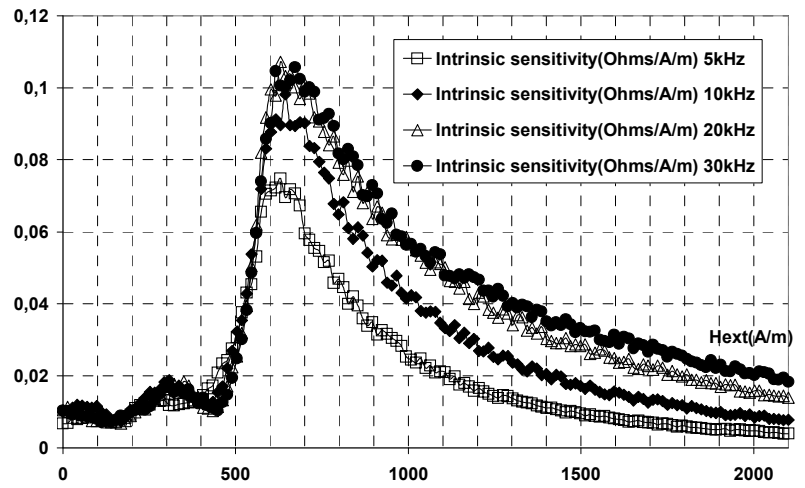

Fig 6: Longitudinal coiled GMI with two ferromagnetic ribbons: Intrinsic sensitivity with DC field at different frequencies.

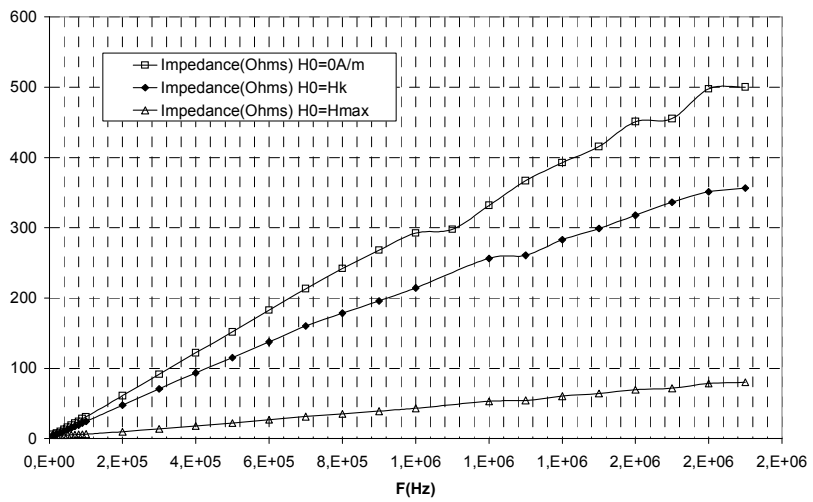

Fig 7: Transverse coiled GMI with one ribbon: Variation of impedance module with frequency at different $D C$ field.



Fig 8: Transverse coiled GMI with one ribbon: Variation of intrinsic sensitivity with frequency at different $D C$ field.
4. PHYSICS MODEL

Qualitative micro magnetism approach

It is usual to investigate magnetic behaviour by the micro magnetic theory. In general, when both static and AC magnetic fields are imposed, the magnetic domain configuration in the ferromagnetic matter is modified due to magnetization rotation [8] and the domain wall movement mechanism. But in the simple case of the transverse coil specimen, only uniform rotation appears because of orthogonal direction of the $\mathrm{AC}$ and $\mathrm{DC}$ field to the anisotropic magnetic field. Considering an ideal transverse domain micro-structure, the solutions of the susceptibility tensor are given by the Landau-LifshitzGilbert equation (2) where $\gamma$ corresponds to the gyromagnetic ratio, $\alpha$ traduces the damping phenomenon and $M_{s}$ is the saturated value of magnetization of one domain.

$$
\frac{d \vec{M}}{d t}=-\gamma \mu_{0} \vec{M} \times \vec{H}_{e f f}+\frac{\alpha}{M_{s}} \vec{M} \times \frac{d \vec{M}}{d t}
$$

The effective magnetic field $H_{\text {eff }}(4)$ is deduced from the minimizing of the free energy (3) which is the sum of magnetic anisotropy energy and the external field energy.

$$
\begin{aligned}
& U=k_{u} \sin ^{2} \theta-\mu_{0} M_{s} \sin (\theta)\left(H_{0}+h_{e x}\right) \\
& \vec{H}_{e f f}=-\frac{1}{\gamma \mu_{0} M_{s}} \frac{\partial U}{\partial \theta}
\end{aligned}
$$

When the small alternating magnetic excitation $h_{e x}$ created by $I_{e x c}$ is imposed, a linear solution of (2) can be obtained with respect to the DC field $H_{0}$. In order to determine the former solution, equation (3) must be resolved with the equilibrium conditions of magnetization vector and the rotation angle $\theta$ is therefore given by the approximation relation (5) where $k_{\theta}$ is a fit parameter. Then magnetization $M$ rotates until it is parallel to $H_{0}$ without a singular point at the $H_{k}$ value.

$$
\theta=\frac{\pi}{2} \tanh \left(k_{\theta} \frac{H_{0}}{H_{k}}\right)
$$

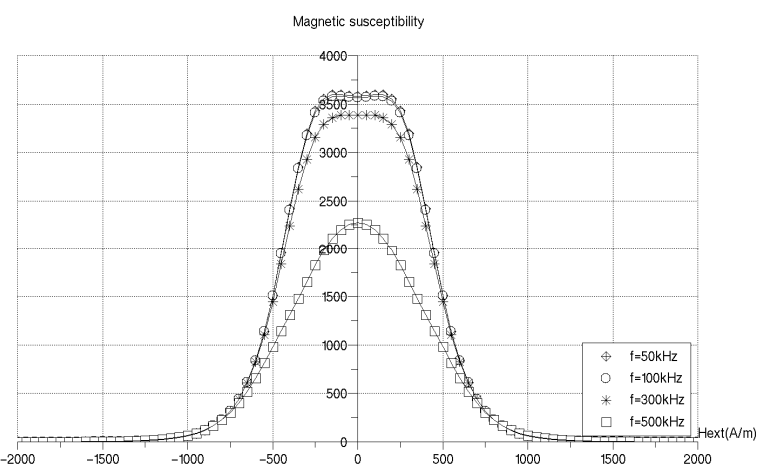

Fig 9: Magnetic susceptibility $\chi_{z z}$ with DC field at different frequencies

Figure 9 above represents the $\chi_{\text {zz }}$ component susceptibility which has been calculated with these following fitting 
parameters: $\gamma=2 \mathrm{e} 10 \mathrm{~m} \cdot \mathrm{A}^{-1} \mathrm{~s}^{-1} ; \alpha=2 ; \mathrm{M}_{\mathrm{s}}=2 \mathrm{e} 6 \quad \mathrm{~A} \cdot \mathrm{m}^{-1}$ and $\mathrm{H}_{\mathrm{k}}=557 \mathrm{~A} \cdot \mathrm{m}^{-1}$. At zero DC field the susceptibility is equal to its initial value. When the DC field reaches the magnetic anisotropic field, the susceptibility strongly decreases and becomes null.

\section{Differential magnetic permeability}

Last results have shown magnetization rotation susceptibility principle which can be determined by differential magnetic permeability measurements. Thus, from experimental results (Figure 3), magnetic permeability can be represented by expression (6) where $H_{e x t}, H_{k}, \mu_{y y i}, \mu_{y y S a t}, \omega$ and $\omega_{0}$ are respectively, the DC field, the anisotropic magnetic field, the initial and the saturated effective permeability, the current frequency and the relaxation frequency. And $S_{\mu}$ is the fitting slope parameter.

$$
\mu_{y y}=-\frac{\left(0.5 *\left(-\mu_{y y i}+\mu_{y y S a t}\right)\right)\left(\tanh \left(S_{\mu}\left(\left|H_{e x t}\right|-H_{k}\right)\right)+1\right)+\mu_{y y i}}{\sqrt{\left(1+\frac{\omega^{2}}{\omega_{0}^{2}}\right)}}
$$

\section{Transverse coiled impedance and sensitivity}

The impedance is deduced from the circulation of the electric field along the coil by considering the homogenous magnetization of the matter. Here in equation (7): $N_{b}$ is the number of turns; $R_{b}$ is the electric resistance of the coil, and parameters $d, e_{p}$ and $L$ are respectively the width, the thickness and the length of a ribbon. The penetration depth is illustrated by equation (8).

$$
\underline{Z}=R_{b}+j \omega\left(\frac{N_{b}^{2} \mu_{y y} d e_{p}}{L(1+j) \frac{e_{p}}{2 \delta}} \tanh \left((1+j) \frac{e_{p}}{2 \delta}\right)\right)
$$

$$
\delta=\sqrt{2 /\left(\sigma \mu_{y y} \omega\right)}
$$

Consequently, the intrinsic sensitivity relation (9) is calculated from equation (7) in the case of low frequencies.

$$
S_{\mathrm{int}}=\frac{\partial \underline{Z}}{\partial H_{0}}=\frac{j \omega}{\sqrt{\left(1+\frac{\omega^{2}}{\omega_{0}^{2}}\right)}} \frac{N_{b}^{2} d e_{p} S_{\mu}\left(\mu_{y y S a t}-\mu_{y y i}\right)}{2 L \cdot \cosh \left(S_{\mu}\left(H_{e x t}-H_{k}\right)\right)^{2}}
$$

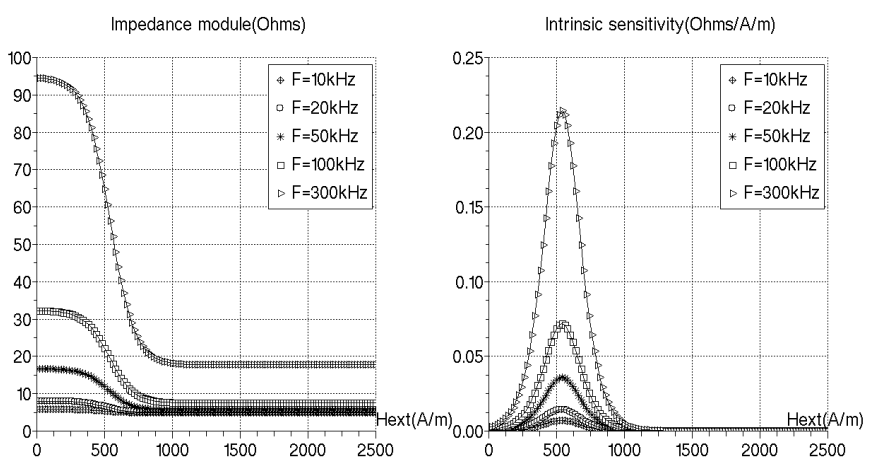

Fig 10: Theoretical results of the transverse coiled GMI with one ribbon: Impedance and intrinsic sensitivity.

Theoretical impedance and intrinsic sensitivity are plotted on Figure 10. The curves exhibit a good accuracy compared to the experimental results on Figure 3 and Figure 5. Nevertheless, above $300 \mathrm{kHz}$ the magnetic permeability frequency behaviour requires more studies.

\section{CONCLUSION}

Sensor performances and physics basis of new GMI transducers have been investigated and have permitted to obtain high sensitive behaviour to weak DC magnetic field. These Coiled GMI elements can easily be made and integrated in demodulation amplitude electronic processing. Sensitivity is directly related to the derivative of the differential magnetic permeability which strongly varying at the anisotropic magnetic field. That was confirmed by using the micro magnetic approach in the case of the transverse type. The results have shown that intrinsic sensitivity can be more than 15 times higher than that of GMI sandwiches. These transducers have been studied for high sensitive space magnetometers which require robustness, low mass and low power consumption.

\section{REFERENCES}

[1] E.P. Harrison, G.L Turney, H. Rowe, H. Gollop, "The electrical properties of High permeability Wires Carrying Alternating Current", Proc. Royal. Soc, Vol $157,1936$.

[2] K. Mohri, L.V Panina, "High frequency Giant Magneto-Impedance in Co-Rich Amorphous Wires and Films", J. Magn. Soc. Japan., Vol. 19, №2, 1995.

[3] N. Fry et al, "Off-Diagonal Magnetoimpedance in $\mathrm{NiFe}-\mathrm{Au}-\mathrm{NiFe}$ Layered Film and its Application to Linear Magnetic Sensors", IEEE Trans. Magn, Vol. 40, Sept. 2004.

[4] B. Kaviraj, S.K. Ghatak, "Very large magnetoimpedance and its scaling behaviour in amorphous $\mathrm{Fe}_{73.5} \mathrm{NB}_{3} \mathrm{Cu}_{1} \mathrm{Si}_{13.5} \mathrm{~B}_{9}$ ribbon", Physica B 403 (2008) 1731.

[5] J. Moutoussamy et al.,"Feasibility of a giant magnetoImpedance sandwich magnetometer for space applications", IEEE Sensors Conf. Atlanta, USA, pp 1013-1016, Oct. 2007.

[6] R.M Bozorth, Ed., Ferromagnetism., New York, IEEE Press, 1993.

[7] A. Qasimi et al., "Improvements of the $\mu$-Hall magnetic sensor: Sensitivity at low frequency", IEEE Sensors Journal, Vol 4, n¹, pp 160-166, February 2004.

[8] C.Dong, S.C. Chen, T.Y. Hsu, "A simple model of giant magnetoimpedance effect in amorphous thin films", J. Magn. Magn. Mater, 250, 2002, pp 633-635.

\section{CONTACT}

* J. Moutoussamy, tel: +33 01-39-25-48-58;

joel.moutoussamy@1pp.polytechnique.fr 